\title{
LEISHMANIOSE VISCERAL NA REGIÃO DE SOBRAL-CE: PERFIL EPIDEMIOLÓGICO DOS CASOS NOTIFICADOS ENTRE OS ANOS DE 2015 A 2018
}

VISCERAL LEISHMANIOSIS IN THE REGION OF SOBRAL-CE: EPIDEMIOLOGICAL PROFILE OF CASES NOTIFIED BETWEEN 2015-2018

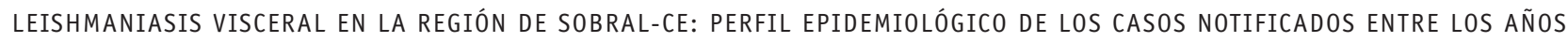
DE 2015 HASTA 2018

Cynira Kezia Rodrigues Ponte Sampaio ${ }^{1}$ Inara Pereira da Cunha 2

Jaqueline Vilela Bulgareli 3

Luciane Miranda Guerra 4

Brunna Verna Castro Gondinho 5

Karine Laura Cortellazzi 6

Como Citar: Sampaio CKRP, Cunha IP, Bulgareli JV, Guerra LM, Gondinho BVC, Cortellazzi KL. Leishmaniose visceral na região de Sobral-CE: perfil epidemiológico dos casos notificados entre os anos de 2015

a 2018. Sanare (Sobral, Online). 2021;20(1):7-16.

Palavras-chave: Leishmaniose visceral; Epidemiologia descritiva; Sistemas de informação.

Keywords: Visceral Leishmaniasis; Descriptive Epidemiology; Information Systems.

Palabras clave: Leishmaniasis visceral; Epidemiología descriptiva; Sistemas de información.

Submetido: $02 / 02 / 2021$

Aprovado:

$14 / 06 / 2021$

Autor(a) para Correspondência: Inara Pereira da Cunha E-mail: inara-pereira@hotmail.com

\section{RESUMO}

o objetivo do estudo foi descrever o perfil epidemiológico dos casos de leishmaniose visceral (LV) na região de saúde de Sobral, Ceará, entre 2015 e 2018. Tratou-se de estudo descritivo com dados das fichas de notificação compulsória registradas no Sistema de Informações de Agravos de Notificação. Entre os anos estudados foram notificados 247 casos de LV. 0 coeficiente de incidência diminuiu de 11,91 por 100.000 habitantes, no ano de 2015, para 7,05, no ano de 2018. Houve maior incidência de LV entre pacientes do sexo masculino (63,16\%), pardos $(88,26 \%)$, de 0 a 4 anos (43,72\%), com menor nível de escolaridade (10,93\%), residentes na zona rural (51,42\%), na microrregião de Sobral (46,15\%). Verificou-se que 54,66\% dos casos evoluíram para cura e 4,05\% para óbito. Conclui-se que ainda é alta a incidência de LV na região de saúde de Sobral, de modo que ações públicas para o enfrentamento da doença devem ser realizadas considerando o perfil identificado.

1. Medicina Veterinária. Mestra em Gestão e Saúde Coletiva. Centro Universitário UNINTA. E-mail: ckrpsampaio@ hotmail.com; ORCID: https://orcid.org/0000-0002-6270-1959

2. Odontologia. Doutorado em Odontologia. Escola de Saúde Pública Dr. Jorge David Nasser. E-mail: inara-pereira@ hotmail.com; ORCID: https://orcid.org/0000-0002-5330-6869

3. Odontologia. Pós-Doutorado em Odontologia. Faculdade de Odontologia. Universidade Federal de Uberlândia. E-mail: jaquelinebulgareli@gmail.com; ORCID: https://orcid.org/0000-0001-7810-0595

4. Odontologia. Pós-Doutorado em Odontologia. Faculdade de Odontologia de Piracicaba. Universidade Estadual de Campinas. E-mail: lumiranda1302@gmail.com; 0RCID: https://orcid.org/0000-0002-7542-7717

5. Odontologia. Doutorado em Odontologia. Faculdade de Odontologia e Enfermagem. Universidade Estadual do Piauí. E-mail: bvernagondim@hotmail.com; ORCID: https://orcid.org/0000-0002-1061-4407

6. Odontologia. Pós-Doutorado em Odontologia. Faculdade de Odontologia de Piracicaba. Universidade Estadual de Campinas. E-mail: karinela@unicamp.br; ORCID: https://orcid.org/0000-0001-9584-9477

Cert. de Redação Científica: Central das Revisões. Edição de texto: Karina Maria M. Machado. Revisão de provas: Texto definitivo validado pelos(as) autores(as). 


\section{ABSTRACT}

The aim of the study was to describe the epidemiological profile of cases of Visceral Leishmaniasis (VL) in the health region of Sobral/Ceará (CE), between 2015 and 2018. This was a descriptive study with data from compulsory notification forms registered in the Notifiable Diseases Information System. In the years studied, 247 cases of VL were notified. The incidence coefficient decreased from 11.91 per 100,000 inhabitants in 2015 to 7.05 in 2018. There was a higher incidence of VL among male patients (63.16\%), mulattos (88.26\%), from 0 to 4 years old $(43.72 \%)$, with lower educational level (10.93\%), residing in the rural area (51.42\%), in the micro-region of Sobral (46.15\%). We found that $54.66 \%$ of the cases evolved to cure and $4.05 \%$ to death. The conclusion was that the incidence of VL is still high in the health region of Sobral/CE. Public actions to fight the disease must be carried out considering the profile identified.

\section{RESUMEN}

El estudio tiene como objetivo describir el perfil epidemiológico de los casos de Leishmaniasis visceral (LV) en la región sanitaria de Sobral/Ceará (CE), entre 2015 y 2018. Se trató de un estudio descriptivo con los datos de los formularios de notificación compulsoria registradas en el Sistema de Informaciones de Agravios de Notificación. En los años estudiados, fueron notificados 247 casos de LV. El coeficiente de incidencia disminuyó de 11,91 por 100.000 habitantes en el año de 2015, para 7,05 en el año de 2018. Hubo más incidencia de LV entre los pacientes del sexo masculino (63,16\%), pardos (88,26\%), de 0 a 4 años $(43,72 \%)$, con bajo nivel de escolaridad (10,93\%), residentes en la zona rural (51,42\%), en la microrregión de Sobral (46,15\%). Se verificó que 54,66\% de los casos evolucionaron a la cura y 4,05\% para defunción. Se concluye que todavía es alta la incidencia de LV en la región sanitaria de Sobral/CE. Acciones públicas para el enfrentamiento de la enfermedad deben ser realizadas considerando el perfil identificado.

\section{INTRODUÇÃO}

Leishmania é um gênero de protozoários que parasitam células fagocíticas, especialmente do sistema imunológico, que podem ser transmitidos para cães domésticos e infectar seres humanos por meio da picada de insetos da subfamília dos flebotomíneos ${ }^{1}$. Essa é uma zoonose conhecida como Leishmania Visceral (LV), cujos sintomas iniciais são úlceras na pele, seguidas de febre alta, diminuição dos glóbulos vermelhos e aumento de volume do baço e fígado. Quando não tratada, a doença pode levar a óbito em $90 \%$ casos $^{2}$.

Dada à magnitude do problema, a LV é uma condição que deve ser notificada pelo profissional da área da saúde, diante da suspeita ou confirmação do agravo, sendo registrado no Sistema de Informação de Agravos de Notificação (SINAN). Essas informações registradas são disponibilizadas à população por meio do acesso à internet e tornam-se de fundamental importância para traçar perfis epidemiológicos de agravos, detectando surtos, epidemias e apontando para estratégias de enfrentamento ${ }^{3}$.
De acordo com a Organização Pan-Americana de Saúde (OPAS), foram reportados 59.769 casos novos nos países da América, resultando em uma média de 3.516 casos por ano entre os anos de 2001 a 2017. 0 Brasil notificou nesse período 96\% (57.582) de novos casos, à frente dos países Sul Americanos como Argentina, Colômbia, Paraguai e Venezuela ${ }^{4}$. Ainda, o Ministério da Saúde (MS) informa que 3.500 casos são registrados anualmente no país e o coeficiente de incidência é de 2 casos a cada 100.000 habitantes. Nos últimos anos, a letalidade aumentou de 3,1\% em 2000 para $7,1 \%$ em $2012^{5}$.

É predominante a endemia da LV no Nordeste do Brasil. Nessa região, o Ceará descreve casos desde a década de 1930. De 2008 a 2018, o estado registrou 6.946 novos casos, com média anual de 534 registrados, incidência média de 6,7 casos por 100.000 habitantes e 414 óbitos $^{6}$. Nesse contexto, a região de Sobral, interior do Ceará, registra desde o ano de 2002 casos de $L V,{ }^{7}$ e foi o cenário do primeiro surto epidêmico da doença no país ${ }^{8}$, despertando, desde então, a necessidade de investigações epidemiológicas na regiã $0^{7,9}$. 
Desde a sua classificação como área de transmissão intensa de LV, Sobral realiza ações de enfrentamento que culminaram com a aparente diminuição da doença ao longo dos anos, de 173 novos casos, entre 2001 e 20107, para 61 casos entre os anos de 2011 a 2015². Porém, Sobral compõe, com outros municípios, a região de saúde de Sobral, apresentando peculiaridades ambientais, climáticas, geográficas, urbanas e culturais que favorecem a existência e propagação de vetores de várias doenças, dentre os quais, os flebotomíneos ${ }^{10}$. Dessa forma, também é importante considerar a região de saúde como campo de investigação do endemismo da doença.

Visando contribuir para o conhecimento acerca das características epidemiológicas da LV e embasar o planejamento das ações no âmbito da vigilância e no controle da doença, o presente estudo teve como objetivo descrever o perfil epidemiológico da leishmaniose visceral na região de saúde de Sobral, região Norte do estado do Ceará, por meio dos casos notificados no período de 2015 a 2018.

\section{METODOLOGIA}

Trata-se de estudo descritivo-exploratório com todos os dados secundários notificados de LV na região de Sobral, Ceará, registrados do Sistema Nacional de Agravos de Notificação ${ }^{11}$ no período de 2015 a 2018.

0 presente estudo considerou como campo de investigação a região de saúde de Sobral, organizada a partir do princípio da regionalização do Sistema Único de Saúde (SUS). No estado do Ceará, a regionalização em saúde, período da coleta, estava organizada em 22 microrregiões de saúde e 3 macrorregiões de saúde (Sobral, Fortaleza e (ariri) ${ }^{12}$.

À época, a região de saúde de Sobral, ou macroregião de Sobral, era composta pelas microrregiões de Sobral, Acaraú, Tianguá, Crateús e Camocim, que totalizam 55 municípios. A microrregião de Sobral situa-se ao norte e é formada por 24 municípios, configurando-se na maior coordenadoria em relação ao número de cidades. A região de saúde de Sobral possui uma estimativa populacional de 638.065 habitantes, tendo 201.756 habitantes o município com maior população (Sobral) ${ }^{12}$. A área territorial da região apresenta $17.333,51 \mathrm{~km}^{213}$.

Os dados sobre as notificações de LV da região de saúde de Sobral estavam disponíveis no programa TABNET do departamento de informática do Sistema Único de Saúde do Brasil ${ }^{11}$. Os mesmos foram transcritos para o programa Microsoft Excel e tabulados para posterior análise. Nessa etapa, foram calculadas as frequências e porcentagens das seguintes variáveis dos usuários acometidos por LV: sexo, faixa etária, raça, nível de escolaridade, tipo de moradia e local de residência. As características da doença também foram tabuladas e descritas (tipo de entrada no sistema de saúde, critério de confirmação do diagnóstico, coinfecção por HIV e evolução do caso).

Realizou-se análise descritiva por meio de tabelas de frequência e porcentagem, com o auxílio do Microsoft Office Excel 2010. As estimativas populacionais empregadas para o cálculo do coeficiente de incidência por 100.000 habitantes foram obtidas do Instituto Brasileiro de Geografia e Estatística (IBGE), considerando a população estimada de 638.065 habitantes na região de saúde de Sobral ${ }^{14}$.

Os municípios brasileiros podem ser divididos em três classes de transmissão de LV, dependendo do número de notificações nos últimos anos. Os municípios com média de casos menor que 2,4 são classificados como de transmissão esporádica; os municípios com média de casos maior ou igual a 2,4 são classificados como transmissão moderada; e aqueles que estão com média de casos maior ou igual a 4,4 são classificados como de transmissão intensa ${ }^{2}$. Assim, para a classificação de transmissão de LV nos municípios da região de saúde de SobralCE, foram utilizados esses critérios mencionados.

0 estudo foi aprovado pelo Comitê de Ética em Pesquisa da Faculdade de 0 dontologia de Piracicaba, da Universidade Estadual de Campinas (FOPUnicamp), de acordo com as normas estabelecidas pela Resolução n. ${ }^{\circ} 466 / 2012$, do Conselho Nacional de Saúde, sob parecer número 3.094.700/2018.

\section{RESULTADOS}

No período de 2015 a 2018, foram notificados 247 dos casos de LV na região de Sobral. Na Figura 1, apresenta-se o número de casos e o coeficiente de incidência de leishmaniose visceral notificados na região, no período de 2015 a 2018. 
Figura 1. Número de casos e coeficiente de incidência de leishmaniose visceral notificados na região de saúde de Sobral-CE, no período de 2015 a 2018.

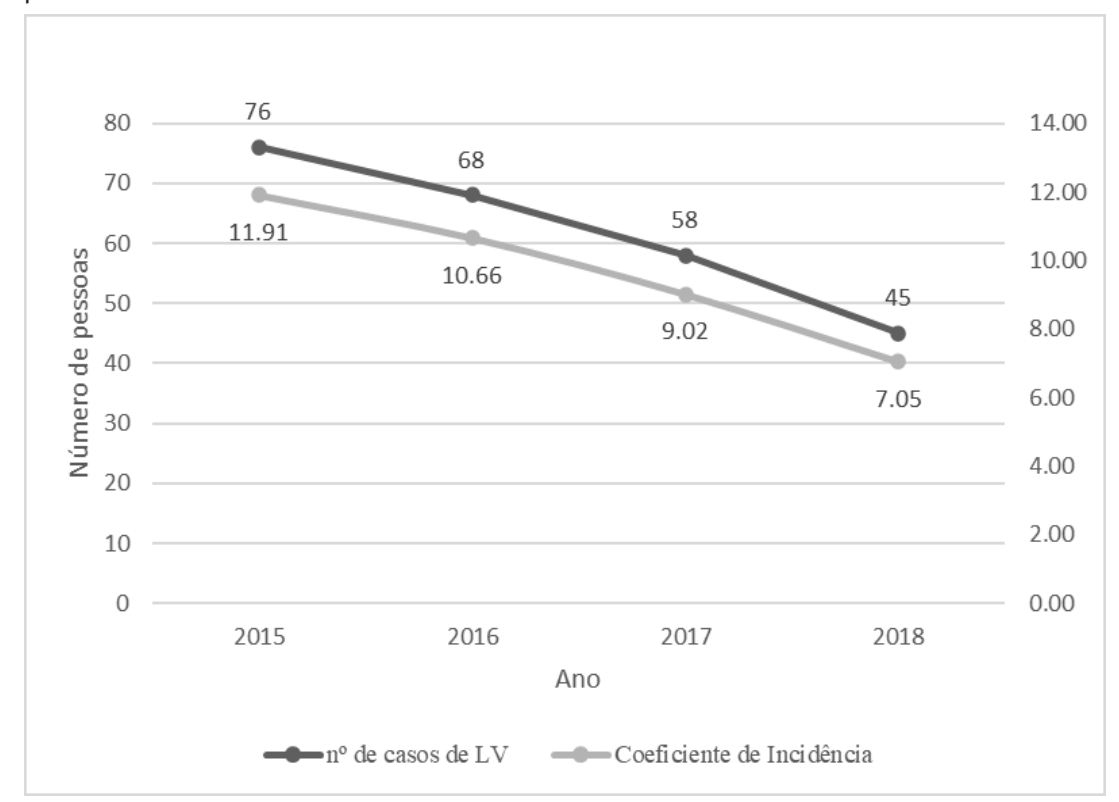

Fonte: Ministério da Saúde/SVS, SINAN Net ${ }^{11}$.

0 coeficiente de incidência e o número de casos registrados nesse período mostram uma tendência linear decrescente dos casos: de 11,91 no ano de 2015 para 7,05 no ano de 2018.

Já na Tabela 1, têm-se as características demográficas dos casos de leishmaniose visceral notificados na Região de Sobral, no período estudado.

Tabela 1. Características demográficas dos casos de leishmaniose visceral notificados na região de saúde de Sobral-CE, no período de 2015 a 2018.

\begin{tabular}{|c|c|c|c|c|c|}
\hline \multirow[t]{2}{*}{ Características } & $\begin{array}{c}2015 \\
(N=76)\end{array}$ & $\begin{array}{c}2016 \\
(N=68)\end{array}$ & $\begin{array}{c}2017 \\
(N=58)\end{array}$ & $\begin{array}{c}2018 \\
(N=45)\end{array}$ & $\begin{array}{c}\text { Total } \\
(\mathrm{N}=247)\end{array}$ \\
\hline & $\mathrm{N}(\%)$ & $\mathrm{N}(\%)$ & $\mathrm{N}(\%)$ & $\mathrm{N}(\%)$ & $\mathrm{N}(\%)$ \\
\hline \multicolumn{6}{|l|}{ Sexo } \\
\hline Masculino & $41(53,95)$ & $45(66,18)$ & $40(68,97)$ & $30(66,67)$ & $156(63,16)$ \\
\hline Feminino & $35(46,05)$ & $23(33,82)$ & $18(31,03)$ & $15(33,33)$ & $91(36,84)$ \\
\hline \multicolumn{6}{|l|}{ Raça/cor } \\
\hline Branca & $2(2,63)$ & $2(2,94)$ & $3(5,17)$ & $0(0,00)$ & $7(2,83)$ \\
\hline Preta & $1(1,32)$ & $5(7,35)$ & $3(5,17)$ & $1(2,22)$ & $10(4,05)$ \\
\hline Parda & $72(94,73)$ & $55(80,89)$ & $50(86,21)$ & $41(91,11)$ & $218(88,26)$ \\
\hline Índio & $0(0,00)$ & $0(0,00)$ & $0(0,00)$ & $1(2,22)$ & $1(0,40)$ \\
\hline Ignorado/Branco & $1(1,32)$ & $6(8,82)$ & $2(3,45)$ & $2(4,44)$ & $11(4,45)$ \\
\hline \multicolumn{6}{|c|}{ Faixa etária (em anos) } \\
\hline $0-4$ & $41(53,95)$ & $23(33,82)$ & $28(48,28)$ & $16(35,56)$ & $108(43,72)$ \\
\hline $5-14$ & $10(13,16)$ & $12(17,65)$ & $8(13,79)$ & $6(13,33)$ & $36(14,57)$ \\
\hline $15-19$ & $3(3,95)$ & $5(7,35)$ & $4(6,90)$ & $2(4,44)$ & $14(5,67)$ \\
\hline $20-39$ & $13(17,10)$ & $17(25,00)$ & $7(12,07)$ & $12(26,68)$ & $49(19,84)$ \\
\hline $40-59$ & $6(7,89)$ & $6(8,83)$ & $9(15,52)$ & $5(11,11)$ & $26(10,53)$ \\
\hline $60-64$ & $1(1,32)$ & $0(0,00)$ & $0(0,00)$ & $1(2,22)$ & $2(0,81)$ \\
\hline $65-69$ & $2(2,63)$ & $2(2,94)$ & $1(1,72)$ & $1(2,22)$ & $6(2,43)$ \\
\hline$>69$ anos & $0(0,00)$ & $3(4,41)$ & $1(1,72)$ & $2(4,44)$ & $6(2,43)$ \\
\hline
\end{tabular}




\begin{tabular}{|c|c|c|c|c|c|}
\hline \multirow[t]{2}{*}{ Características } & $\begin{array}{c}2015 \\
(N=76)\end{array}$ & $\begin{array}{c}2016 \\
(N=68)\end{array}$ & $\begin{array}{c}2017 \\
(N=58)\end{array}$ & $\begin{array}{c}2018 \\
(N=45)\end{array}$ & $\begin{array}{c}\text { Total } \\
(\mathrm{N}=247)\end{array}$ \\
\hline & $\mathrm{N}(\%)$ & $\mathrm{N}(\%)$ & $\mathrm{N}(\%)$ & $\mathrm{N}(\%)$ & $\mathrm{N}(\%)$ \\
\hline \multicolumn{6}{|l|}{ Escolaridade } \\
\hline Analfabeto & $0(0,00)$ & $0(0,00)$ & $3(5,17)$ & $1(2,22)$ & $4(1,62)$ \\
\hline $1^{\mathrm{a}}$ a $4^{\mathrm{a}}$ série incompleta & $0(0,00)$ & $0(0,00)$ & $6(10,34)$ & $2(4,44)$ & $8(3,24)$ \\
\hline $4^{\mathrm{a}}$ série completa & $2(2,63)$ & $2(2,94)$ & $0(0,00)$ & $0(0,00)$ & $4(1,62)$ \\
\hline $5^{\mathrm{a}}$ a $8^{\mathrm{a}}$ série incompleta & $6(7,89)$ & $10(14,71)$ & $7(12,07)$ & $4(8,89)$ & $27(10,93)$ \\
\hline Ensino fundamental completo & $0(0,00)$ & $1(1,47)$ & $0(0,00)$ & $3(6,67)$ & $4(1,62)$ \\
\hline Ensino médio incompleto & $1(1,32)$ & $2(2,94)$ & $2(3,45)$ & $2(4,44)$ & $7(2,83)$ \\
\hline Ensino médio completo & $0(0,00)$ & $2(2,94)$ & $0(0,00)$ & $1(2,22)$ & $3(1,21)$ \\
\hline Não se aplica & $44(57,90)$ & $26(38,24)$ & $30(51,72)$ & $18(40)$ & $118(47,77)$ \\
\hline Ignorado/Branco & $23(30,26)$ & $25(36,76)$ & $10(17,24)$ & $14(31,12)$ & $72(29,15)$ \\
\hline \multicolumn{6}{|l|}{ Tipo de Moradia } \\
\hline Urbana & $30(39,47)$ & $38(55,88)$ & $26(44,83)$ & $19(42,22)$ & $113(45,75)$ \\
\hline Rural & $44(57,90)$ & $29(42,65)$ & $30(51,72)$ & $24(53,34)$ & $127(51,42)$ \\
\hline Ignorado/Branco & $2(2,63)$ & $1(1,47)$ & $2(3,45)$ & $2(4,44)$ & $7(2,83)$ \\
\hline \multicolumn{6}{|l|}{ Localização da residência } \\
\hline Município de Sobral & $14(18,43)$ & $13(19,12)$ & $6(10,34)$ & $7(15,55)$ & $40(16,20)$ \\
\hline $\begin{array}{l}\text { Microrregião de Saúde de } \\
\text { Sobral }\end{array}$ & $33(43,42)$ & $38(55,88)$ & $28(48,27)$ & $15(33,33)$ & $114(46,15)$ \\
\hline $\begin{array}{l}\text { Macrorregião de Saúde de } \\
\text { Sobral }\end{array}$ & $29(38,15)$ & $17(25,00)$ & $24(41,39)$ & $23(51,12)$ & $93(37,65)$ \\
\hline
\end{tabular}

Fonte: Ministério da Saúde/SVS, SINAN Net ${ }^{11}$.

No período estudado, houve predominância do sexo masculino $(63,16 \%)$, a maioria foi classificada como parda $(88,26 \%)$, sendo a maior ocorrência de casos de LV na faixa etária de 0 a 4 anos (43,72\%). Além disso, $21,86 \%$ dos indivíduos tinham nível de escolaridade abaixo do superior completo. De acordo com o tipo de moradia e localização da residência, o maior percentual de casos observado foi na área rural, com um total de $51,42 \%$ de notificações, e na microrregião de Sobral $(46,15 \%)$.

Com relação às notificações na região de saúde de Sobral (macrorregião), observou-se o registro de casos de LV em 45 municípios, sendo 23 deles localizados na macrorregião de Sobral, e 22 municípios na microrregião sobralense. Houve uma média maior que 4,4 casos notificados durante os anos de 2015 a 2018, nos municípios de Sobral (média de $16 \pm 4.08$ ) e Granja (média de 4,5 $\pm 3,11$ ), sendo então classificados como áreas de transmissão intensa de LV. Os municípios com média maior ou igual a 2,4 casos notificados, e classificados como área de

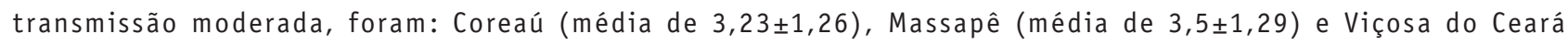
(média de $4 \pm 1,63$ ). Os demais municípios foram classificados como área de transmissão esporádica (média $<2,4$ casos notificados).

$\mathrm{Na}$ Tabela 2, apresentam-se as características clínicas dos casos de leishmaniose visceral notificados na região de saúde de Sobral.

Tabela 2. Características clínicas dos casos de leishmaniose visceral notificados na região de saúde de SobralCE, no período de 2015 a 2018.

\begin{tabular}{lccccc} 
Caracteristicas & $\begin{array}{c}2015 \\
(\mathrm{~N}=76)\end{array}$ & $\begin{array}{c}2016 \\
(\mathrm{~N}=68)\end{array}$ & $\begin{array}{c}2017 \\
(\mathrm{~N}=58)\end{array}$ & $\begin{array}{c}2018 \\
(\mathrm{~N}=45)\end{array}$ & $\begin{array}{c}\text { Total } \\
(\mathrm{N}=247)\end{array}$ \\
\cline { 2 - 6 } & $\mathbf{N}(\%)$ & $\mathbf{N}(\%)$ & $\mathbf{N}(\%)$ & $\mathbf{N}(\%)$ & $\mathbf{N}(\%)$ \\
\hline Tipo de entrada no sistema de saúde & & & & & \\
$\begin{array}{l}\text { Ignorado/Branco } \\
\text { Caso novo }\end{array}$ & $0(0,00)$ & $0(0,00)$ & $2(3,45)$ & $1(2,22)$ & $3(1,21)$ \\
\hline
\end{tabular}




\begin{tabular}{|c|c|c|c|c|c|}
\hline \multirow[t]{2}{*}{ Caracteristicas } & $\begin{array}{c}2015 \\
(N=76)\end{array}$ & $\begin{array}{c}2016 \\
(N=68)\end{array}$ & $\begin{array}{c}2017 \\
(N=58)\end{array}$ & $\begin{array}{c}2018 \\
(N=45)\end{array}$ & $\begin{array}{c}\text { Total } \\
(\mathrm{N}=247)\end{array}$ \\
\hline & $N(\%)$ & $N(\%)$ & $N(\%)$ & $N(\%)$ & $N(\%)$ \\
\hline Recidiva & $1(1,32)$ & $4(5,88)$ & $2(3,45)$ & $2(4,44)$ & $9(3,64)$ \\
\hline Transferência & $21(27,63)$ & $13(19,12)$ & $10(17,24)$ & $8(17,75)$ & $52(21,05)$ \\
\hline \multicolumn{6}{|l|}{ Critério de confirmação } \\
\hline Laboratorial & $74(97,37)$ & $67(98,53)$ & $49(84,48)$ & $35(77,78)$ & $225(91,09)$ \\
\hline Clínico-epidemiológico & $2(2,63)$ & $1(1,47)$ & $9(15,52)$ & $10(22,22)$ & $22(8,91)$ \\
\hline \multicolumn{6}{|l|}{ Coinfecção por HIV } \\
\hline Ignorado/Branco & $12(15,79)$ & $17(25,00)$ & $8(13,79)$ & $9(20,00)$ & $46(18,62)$ \\
\hline Sim & $0(0,00)$ & $3(4,41)$ & $2(3,45)$ & $2(4,44)$ & $7(2,83)$ \\
\hline Não & $64(84,21)$ & $48(70,59)$ & $48(82,76)$ & $34(75,56)$ & $194(78,54)$ \\
\hline \multicolumn{6}{|l|}{ Evolução do caso } \\
\hline Ignorado/Branco & $10(13,16)$ & $14(20,59)$ & $3(5,17)$ & $8(17,78)$ & $35(14,17)$ \\
\hline Cura & $36(47,37)$ & $40(58,82)$ & $28(48,28)$ & $31(68,89)$ & $135(54,66)$ \\
\hline Abandono & $1(1,32)$ & $0(0,00)$ & $0(0,00)$ & $0(0,00)$ & $1(0,40)$ \\
\hline Óbito por leishmaniose visceral & $5(6,58)$ & $1(1,47)$ & $4(6,90)$ & $0(0,00)$ & $10(4,05)$ \\
\hline óbito - outra causa & $0(0,00)$ & $1(1,47)$ & $0(0,00)$ & $1(2,22)$ & $2(0,81)$ \\
\hline Transferência & $24(31,57)$ & $12(17,65)$ & $23(39,65)$ & $5(11,11)$ & $64(25,91)$ \\
\hline
\end{tabular}

Fonte: Ministério da Saúde/SVS, SINAN Net ${ }^{11}$.

Em relação ao tipo de entrada no sistema de saúde, $74,09 \%$ foram casos novos e $91,09 \%$ dos indivíduos tiveram o critério laboratorial como confirmação de diagnóstico, em detrimento do critério exclusivamente clínico-epidemiológico $(8,91 \%)$. Na maioria dos casos não houve coinfecção por HIV $(78,54 \%)$. Ainda, durante o período avaliado, 54,66\% dos casos de LV notificados na região de Sobral evoluíram para a cura, 25,91\% receberam transferência para outro local, 4,05\% evoluíram para o óbito e 1 caso $(0,4 \%)$ abandonou o tratamento.

\section{DISCUSSÃO}

A região de saúde de Sobral, no estado do Ceará, parece apresentar um processo de diminuição endêmica de LV. 0 presente estudo identificou que, na região, houve 247 casos confirmados entre 2012 a 2018; para o mesmo período, houve um decréscimo no coeficiente de incidência de 11,91 para 7,05 novos casos a cada 100.000 habitantes.

Segundo o Ministério da Saúde, foram confirmados, no Brasil, 4.103 casos em 2017, com um coeficiente de incidência de 1,98 novos casos a cada 100.000 habitantes, sendo que $44,5 \%$ foram registrados na Região Nordeste ${ }^{1}$. Tal fato demonstra que a região de saúde de Sobral, quando comparada aos indicadores nacionais, apresenta alta incidência de LV, mesmo com a tendência de diminuição de casos reportados ao SINAN.

Em anos anteriores, o número de notificações no município de Sobral foi menor ${ }^{7,9}$. Observa-se que, nesse município, foram registrados, em 2014, 25 novos casos, número menor do que o registrado em 2011, de 53 notificações ${ }^{9}$. Os resultados presentes reforçam essa tendência decrescente de LV no município de Sobral, pois, durante o período estudado, foi possivel verificar que o número de novos casos diminuiu de 14 para 7 , nos anos de 2015 e 2018, respectivamente.

Entretanto, analisando a LV na perspectiva da região de saúde, observa-se um avanço geográfico da zoonose no período considerado no estudo. Além do município de Sobral ter sido classificado como área de transmissão intensa, a mesma classificação foi observada para o município de Granja, localizado na macrorregião de Sobral. Foram classificadas como áreas de transmissão moderada as cidades de Coreaú, Ipu, Massapê e Viçosa do Ceará. Esses municípios apresentam características demográficas similares, tais como fatores climáticos, temperatura, umidade e pluviometria, e que influenciam na população de flebotomíneos ${ }^{15}$. Além disso, há a correlação da densidade desses vetores com a presença de vegetações (raízes, 
troncos de árvores e matéria orgânica do solo), que podem ser abrigos ou criadouros do vetor ${ }^{10}$.

Destaca-se também que as habitações humanas em lugares inadequados, a construção desordenada de abrigos de animais domésticos e a carência das condições mínimas de saneamento básico são condições comuns em áreas rurais e periféricas de centros urbanos. Essas condições favorecem não apenas a infecção dos animais domésticos, reservatórios de Leishmania, como também a proliferação de flebotomíneos e a propagação da doença em humanos ${ }^{10}$.

Frente a essas condições de vida, que também podem ser encontradas na região de saúde de Sobral, a Secretaria de Saúde do Estado do Ceará implantou estratégias voltadas para o controle da doença, como identificação e eliminação dos reservatórios, dentre eles o cão, aplicação de inseticidas para a eliminação do vetor, diagnóstico e tratamento adequado dos casos registrados. 0 objetivo principal do Programa de LV, na região, é a redução da letalidade por meio do diagnóstico e do tratamento precoce. As ações para o controle da doença envolvem: o tratamento dos casos confirmados; realização de inquérito sorológico canino censitário e capacitação de profissionais para diagnóstico e tratamento da LV6 .

Para tornar as ações de controle da doença ainda mais eficazes, o conhecimento do perfil epidemiológico da LV se faz necessário, uma vez que permite a identificação de grupos vulneráveis ${ }^{16}$. Vale ressaltar que essa caracterização é imperativa para ações de prevenção da doença ${ }^{17}$. Assim, ao identificar o perfil dos casos acometidos, foi observado nos resultados apresentados maior incidência da doença em indivíduos do sexo masculino, corroborando os achados anteriores ${ }^{18}$.

Não há maior susceptibilidade de LV conforme a cor de pele/raça. No entanto, há maior risco da doença de acordo com as condições de renda, que geralmente é menor entre os pardos/negros e na população de menor escolaridade ${ }^{19}$. Há estudos que reportaram que a população de cor parda e de menor nível escolar, por não ter acesso aos bens e serviços básicos, e/ou por não adotar medidas de prevenção à LV, é a mais acometida ${ }^{20-22}$. De acordo com a literatura, os casos de LV entre pardos corresponderam a $79,19 \%$ de uma população estudada no Nordeste?.

Quanto à escolaridade, cabe ainda destacar que o nível de instrução considerado ignorado, em branco ou não se aplica correspondeu a 79,93\% dos achados, isso pode ser um reflexo do número de crianças menores de 4 anos de idade notificadas e que ainda não iniciaram a trajetória escolar, não sendo, desse modo, registradas as informações sobre escolaridade nesse campo da ficha de notificação. Resultado semelhante foi reportado anteriormente no município de Sobral ${ }^{9}$.

Em se tratando da faixa etária, há um maior número de casos de LV no Ceará, entre as crianças menores de 10 anos $^{6}$. Em Sobral, no período de 2011 a 2015, foram notificados 34 casos na faixa de 1 a 4 anos, correspondendo a $23 \%$ dos casos notificados naquele período ${ }^{9}$. Números maiores de notificações foram identificados no presente trabalho. A justificativa para esse achado pode ser analisada pela questão imunológica da criança, que ainda nos primeiros anos de vida está em desenvolvimento. Além disso, há um contato mais frequente das crianças com os animais domésticos ${ }^{23}$, o que pode contribuir com a ocorrência da afecção.

A LV, por tempos, era restrita às áreas rurais, e vem avançando para a periferia dos centros urbanos $^{18}$. Contudo, apesar da urbanização da doença, os casos notificados na zona rural ainda foram maiores na região de saúde de Sobral. Sabese que cães, possíveis reservatórios da parasitose, quando residentes em áreas com maior cobertura de vegetação esparsa, apresentam maior prevalência da infecção do que aqueles que residem em áreas menos vegetadas, sendo esse um fator importante para a proliferação da LV24. Durante o período de 2008 a 2017, 73.964 cães foram testados para a LV no município de Sobral, e 2.833 (3,8\%) foram positivos. No mesmo período, a triagem dos animais foi considerada inconsistente e relativamente baixa, devido à falta de testes sorológicos ${ }^{25}$. Portanto, medidas de prevenção voltadas para a triagem de animais, nas zonas rurais da região de saúde de Sobral, devem ser discutidas no planejamento local.

Dentre os indivíduos com LV no período do estudo, $74,09 \%$ das notificações foram inseridas no sistema de saúde como novos casos; percentual semelhante ao identificado no município de Sobral em anos anteriores $(73,62 \%)^{9}$. Apesar da constante notificação de novos casos, destaca-se a importância do diagnóstico precoce. Foi revelado nos achados presentes que a confirmação da LV por critérios laboratoriais ocorreu em cerca de $90 \%$ dos casos.

0 diagnóstico precoce, por testes laboratoriais, 
aliado à preparação dos profissionais de saúde e uso de medicamentos adequados, contribuem para o aumento da cura da doença ${ }^{9}$. Na região de saúde de Sobral, as taxas de cura da doença e de óbitos demonstram uma possível eficiência do sistema de saúde voltada para a prestação de cuidados aos pacientes acometidos. Essa premissa baseia-se na avaliação dessas taxas em anos anteriores, sendo de $44,11 \%$ e $5,88 \%$, respectivamente, considerando o município de Sobral?.

A LV vem emergindo como doença oportunista em pessoas infectadas com o HIV, bem como em pessoas submetidas a transplantes e em associação com outras condições em que há comprometimento da imunidade ${ }^{26}$. Por isso, a avaliação de casos de coinfecção por HIV nas notificações de LV deve fazer parte do monitoramento da doença. 0 presente estudo identificou que em torno de $78 \%$ dos casos não tiveram coinfecção por HIV, número abaixo do reportado em outras regiões do Nordeste ${ }^{27}$. No estado do Ceará, por exemplo, houve uma média anual, entre 2007 a 2011, de 31,8 8 6,0 indivíduos apresentando coinfecção HIV-LV, com aproximadamente $57,2 \%$ dos casos provenientes do município de Fortaleza ${ }^{18}$. Tal informação aponta para um maior controle dessa condição na região investigada.

Diante dos achados, é possivel constatar que a frequência de LV nos municípios da região de saúde de Sobral, no período de 2015 a 2018, apresentou uma redução no coeficiente de incidência, porém é uma situação considerada distante da meta a ser alcançada para o controle da transmissão da doença. Foram verificados a frequência das notificações em áreas de transmissão intensa (Sobral e Granja) e o perfil da população mais acometida. Portanto, o estudo sinaliza que esses municípios precisam ser averiguados com maior cuidado, sendo preciso o desenvolvimento de ações direcionadas a esse segmento populacional identificado. Sugere-se, assim, disponibilizar estrutura adequada para 0 rastreio e triagem de animais domésticos, debater ações articuladas entre atenção primária em saúde e vigilância em saúde e promover a sensibilização das equipes de saúde no momento dos atendimentos dos casos suspeitos e confirmados.

Outra questão que necessita de destaque é o uso dessas informações para aprimorar as atividades de educação em saúde. A educação em saúde dos sujeitos mais vulneráveis, por meio da incorporação de medidas simples como a limpeza dos quintais, terrenos, eliminação de resíduos e criadouros de vetores, uso de mosquiteiro, telas nas portas e janelas, e o cuidado com a saúde dos animais domésticos, são algumas das estratégias que podem ser aplicadas pela comunidade e que interferem no ciclo de contaminação da $L^{28}$. É fundamental que a população se responsabilize também pelas medidas preventivas e participe ativamente das discussões que visem levantar as possiveis medidas de enfrentamento da LV.

Ressalta-se também que, por se tratar de um estudo transversal, e depender de dados secundários, a pesquisa apresenta algumas limitações, como a possibilidade de subnotificações de LV, bem como incoerências no preenchimento das fichas. No entanto, as informações evidenciadas são pertinentes no planejamento e monitoramento da transmissão da doença, com a finalidade de melhorar o quadro epidemiológico, já que ainda se encontra como um problema de saúde pública na região.

\section{CONCLUSÃO}

0 perfil epidemiológico da região de saúde de Sobral ainda aponta para a endemicidade da doença, com maior concentração de acometidos no sexo masculino, crianças de 0 a 4 anos de idade, raça parda, com baixa escolaridade e residentes na zona rural. Ainda, durante o período estudado, mais da metade dos casos evoluíram para a cura. Apesar da tendência de queda nos valores do coeficiente de incidência e no número de novos casos de LV, ainda se faz necessária a implantação de estratégias de vigilância e prevenção da LV, considerando as características identificadas.

\section{CONTRIBUIÇÃO DAS AUTORAS}

Cynira Kezia Rodrigues Ponte Sampaio e Karine Laura Cortellazzi participaram da concepção e delineamento do estudo, análise e interpretação dos dados; Inara Pereira da Cunha, Jaqueline Vilela Bulgareli, Luciane Miranda Guerra e Brunna Verna Castro Gondinho contribuíram com a redação ou revisão crítica relevante do conteúdo intelectual do manuscrito.

\section{REFERÊNCIAS}

1. Brasil. Secretaria de Vigilância em Saúde Departamento de Vigilância Epidemiológica Coordenação Geral de Doenças Transmissíveis, 
Leishmaniose visceral [document on the internet]. 2017 [cited 2020 Mar 12]. Available from: https://portalarquivos2.saude.gov.br/images/ $\mathrm{pdf} / 2019 /$ janeiro/28/leishvisceral-17-novo-layout. $\mathrm{pdf}$

2. Brasil. Manual de vigilância e controle da leishmaniose visceral. Série A. Normas e Manuais Técnicos. Brasília: Ministério da Saúde; 2016.

3. Barreto ML, Teixeira MG, Bastos FI, Ximenes RAA, Barata RB, Rodrigues LC. Sucessos e fracassos no controle de doenças infecciosas no Brasil: o contexto social e ambiental, políticas, intervenções e necessidades de pesquisa. Lancet. 2011;(3):47-60. doi: https://doi.org/10.1016/ $\underline{\text { S0140-6736(11)60202-X }}$

4. Organização Pan-Americana da Saúde. Informe Epidemiológico das Américas [home-page on the internet]. 2019 [cited 2019 Mar 17]. Available from: https://iris.paho.org/handle/10665.2/51738

5. Brasil. Leishmaniose Visceral: o que é, causas, sintomas, tratamento, diagnóstico e prevenção [home-page on the internet]. Ministério da Saúde; 2017 [cited 2020 Mar 18]. Available from: https:// antigo.saude.gov.br/saude-de-a-z/leishmaniosevisceral

6. Ceará. Boletim epidemiológico: leishmaniose visceral 24 de outubro de 2018 [document on the internet]. Fortaleza: Governo do Estado; 2018 [cited 2020 Mar 18]. Available from: https://www.saude. ce.gov.br/wpcontent/uploads/sites/9/2018/06/ boletim leishimaniose $24 \quad 10$ 2018.pdf

7. Oliveira LS, Neto RVD, Braga PET. Perfil epidemiológico dos casos de leishmaniose visceral em Sobral, Ceará no período de 2001 a 2010. Sanare (Sobral, 0nline) [serial on the internet]. 2018 [cited 2020 Mar 18];17(1):21-57. Available from: https://sanare.emnuvens.com.br/sanare/article/ view $/ 323$

8. Lainson R, Rangel EF. Lutzomyia longipalpis and the eco-epidemiology of American visceral leishmaniasis, with particular reference to Brazil - A Review. Memória do Instituto Oswaldo Cruz. $2005 ; 100(1): 811-27$.

9. Sousa NA, Linhares CB, Pires FGB, Teixeira TC, Lima JS, Nascimento MLO. Perfil epidemiológico dos casos de leishmaniose visceral em Sobral-CE de 2011 a 2015. Sanare (Sobral, Online) [serial on the internet]. 2018 [cited 2020 Mar 18];17(1):517. Available from: https://sanare.emnuvens .com.br/sanare/article/view/1222

10. Macedo ITF, Bevilaqua CML, Morais NB, Sousa LC, Linhares FE, Amóra SSA, et al. Sazonalidade de flebotomíneos em área endêmica de leishmaniose visceral no município de Sobral, Ceará, Brasil. Ciênc anim. 2008;18(2):67-74.

11. Sistema de Informação de Agravos de Notificação Sinan [home-page on the internet]. 2018 [cited 2019 Dec 20]. Available from: https://portalsinan. saude.gov.br/

12. Ceará. Regionalização da Saúde [home-page on the internet]; 2018 [cited 2020 Mar 18]. Available from: https://www.saude.ce.gov.br/2008/09/09/ unidades-regionais-de-saude/

13. Instituto de Pesquisa e Estratégia Econômica do Ceará. Perfil das Regiões de Planejamento [homepage on the internet]. 2017 [cited 2020 Mar 21]. Available from: https://www.ipece.ce.gov.br/ regioes-de-planejamento/

14. Instituto Brasileiro de Geografia e Estatística. Censo demográfico [home-page on the internet]. 2010 [citado 2020 Mar 18]. Available from: https:// cidades.ibge.gov.br/?codmun $=231290$

15. Silvino AS, Silva KB, Sousa-Paula LC, Dias RD, Braga PET. Caracterização de flebotomíneos em bairros de Sobral, Ceará. Revista da Biologia. 2017;12(17). doi: https://doi.org/10.759 4/revbio.17.02.04

16. Paim JS. Epidemiologia e planejamento: a recomposição das práticas epidemiológicas na gestão do SUS. Ciênc Saúde Colet. 2003;8(2):55767. doi: https://doi.org/10.1590/S1413$\underline{81232003000200017}$

17. Barbosa MN, Guimarães EAZ, Luz ZMP. Avaliação de estratégia de organização de serviços de saúde para prevenção e controle da leishmaniose visceral. Epidemiol Serv Saúde. 2016;25(3):56374. doi: https://doi.org/10.5123/s167949742016000300012

18. Cavalcante IJM, Vale MR. Aspectos epidemiológicos da leishmaniose visceral (calazar) no Ceará no período de 2007 a 2011. Rev Bras Epidemiol. 2014;17(4):911-24. doi: https://doi. org/10.1590/1809-4503201400040010

19. Gomes MRS, Solange CI. Assimetrias salariais de gênero e a abordagem regional no brasil: uma análise segundo a admissão no emprego e setores de atividade. Rev econ contemp. 2018;22(3): e182234. doi: https://doi.org/10.1590/198055272234

20. Farias HMT, Gusmão JF, Aguilar RV, Barbosa SFA. Perfil epidemiológico da leishmaniose visceral humana nas regiões de saúde do norte de minas gerais. Enf foco. 2019;10(2):90-96. doi: https:// doi.org/10.21675/2357-707X.2019.v10.n2.1887

21. Silva-Filho AG, Carmo DM, Marques AS, Afonso MPD, Oliveira SV. Situação epidemiológica das 
leishmanioses em Uberlândia, Minas Gerais. Rev Saúde Coletiva da UFES. 2019;9(1):166-72. doi: https://doi.org/10.13102/rscdauefs.v9i0.4435

22. Menezes JA, Luz TCB, Sousa FF, Verne RN, Lima FP Margonari C. Fatores de risco peridomiciliares e conhecimento sobre leishmaniose visceral da população de Formiga, Minas Gerais. Rev Bras Epidemiol. 2016;19(2):362-74. doi: https://doi. org/10.1590/1980-5497201600020013

23. Queiroz MJA, Alves JGB, Correia JB. Leishmaniose visceral: características clínico-epidemiológicas em crianças de área endêmica. J Pediatr. 2004;80(2):141-46. doi: https://doi.org/10.1590/ S0021-75572004000200012

24. Rotti AT, Werneck GL, Almeida AS, Figueiredo FB. Fatores ambientais associados à ocorrência de leishmaniose visceral canina em uma área de recente introdução da doença no Estado do Rio de Janeiro, Brasil. Cad Saúde Pública. 2018;34(1):e00021117. doi: https://doi.org/10.1590/0102-311×00021117

25. Sousa-Paula LC, Silva LGD, Sales KGDS, DantasTorres F. Failure of the dog culling strategy in controlling human visceral leishmaniasis in Brazil: A screening coverage issue? PLoS Negl Trop Dis. 2019;13(6):e0007553. doi: https://doi. org/10.1371/journal.pntd.0007553

26. Sousa RLT, Nunes MI, Freire S.M. Perfil epidemiológico de pacientes com leishmaniose visceral notificados em hospital de referência em Teresina-PI. RIES. 2019;8(1):126-35.

27. Carvalho FL, Aires DLS, Segunda ZF, Azevedo ZMP, Corrêa RGCF, Aquino DMC, et al. Perfil epidemiológico dos indivíduos HIV positivo e coinfecção HIV-Leishmania em um serviço de referência em São Luís, MA, Brasil. Ciênc Saúde Colet. 2013;18(5):1305-12. doi: https://doi. org/10.1590/S1413-81232013000500015

28. Martins $C P$, Brandão MGSA, Braga $M M$, Sampaio LBF, Barros LM, Pacheco JCB. Monitoramento epidemiológico como instrumento de apoio à gestão de saúde: análise das notificações de leishmaniose visceral em Sobral, Ceará. Rev Adm Saúde. 2018;18(72). doi: https://doi.org/10.23973/ $\underline{\text { ras. } 72.117}$
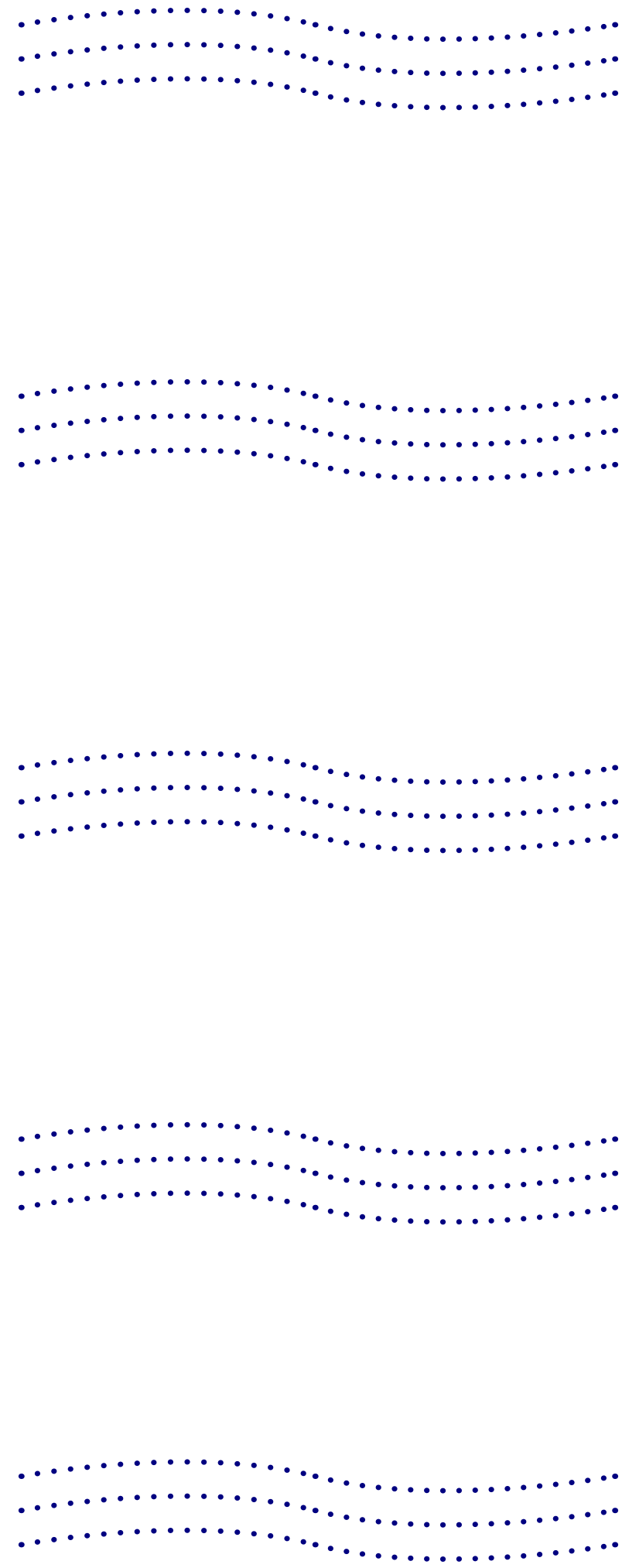